\title{
BLOW-UP PROPERTIES FOR PARABOLIC SYSTEMS WITH LOCALIZED NONLINEAR SOURCE
}

\author{
JuN ZHOU
}

Abstract. This paper deals with blow-up properties of solutions to a semilinear parabolic system with nonlinear localized source involved a product with local terms

$$
u_{t}=\Delta u+\exp \left\{m u(x, t)+n v\left(x_{0}, t\right)\right\}, \quad v_{t}=\Delta v+\exp \left\{p u\left(x_{0}, t\right)+q v(x, t)\right\}
$$

with homogeneous Dirichlet boundary conditions. We investigate the influence of localized sources and local terms on blow-up properties for this system, and prove that: $(i)$ when $m, q \leqslant$ 0 this system possesses uniform blow-up profiles, in other words, the localized terms play a leading role in the blow-up profile for this case; (ii) when $m, q>0$, this system presents single point blow-up patterns, or say that local terms dominate localized terms in the blow-up profile. Moreover, the blow-up rate estimates in time and space are obtained, respectively.

Mathematics subject classification (2000): 35B40, 35K65, 35K15.

Keywords and phrases: parabolic system, nonlinear localized source, nonlinear local terms, uniform blow-up profile, single point blow-up pattern, blow-up rate estimate.

\section{REFERENCES}

[1] K. Bimpong-Bota, P. Ortoleva And J. Rossi, Far-from-equilibrium phenomena at local sites of reaction, J. Chem. Phys. 60 (8) (1974), 3124-3133.

[2] J. R. CANNON AND H. M. Yin, A class of non-linear non-classical parabolic equations, J. Diff. Equations 79 (1989), 266-288.

[3] J. M. ChadAm, A. Pierce And H. M. Yin, The blow-up properties of solutions to some diffusion equations with localized nonlinear reactions, J. Math. Anal. Appl. 169 (1992), 313-328.

[4] M. Escobedo And M. A. Herrero, A semilinear parabolic system in a bounded domain, Annali di Matematica pura ed applicata CLXV (IV) (1993), 315-336.

[5] A. FRIEDMAN AND J. B. MCLEOD, Blow-up of positive solutions of semilinear heat equation, Indiana Univ. Math. J. 34 (2) (1985), 425-447.

[6] V. A. Galaktionov And J. L. VÁZQuez, The problem of blow-up in nonlinear parabolic equation, Discrete and Continuous Dynamical System 8 (2) (2002), 399-433.

[7] F. C. Li, S. X. HuAng AND C. H. XIE, Global existence and blow-up of solutions to a nonlocal reaction-diffusion system, Discrete and Continuous Dynamical System 9 (6) (2003), 1519-1523.

[8] H. L. Li AND M. X. WANG, Blow-up properties for parabolic systems with localized nonlinear sources, Appl. Math. Letters 17 (2004), 771-778.

[9] H. L. LI AND M. X. WANG, Uniform blow-up profiles and boundary layer for a parabolic system with localized nonlinear reaction terms, Science in China, series A 48 (2) (2005), 185-197.

[10] H. L. LI AND M. X. WANG, Properties of blow-up solutions to a parabolic system with nonlinear localized terms, Discrete and Continuous Dynamical System 13 (3) (2005), 683-700.

[11] Z. G. Lin, C. H. XIE AND M. X. WANG, The blow-up properties of solutions to a parabolic system with localized nonlinear reactions, Acta Mathematica Scientia 18 (4) (1997), 413-420.

[12] P. ORTOLEVA AND J. Rossi, Local structures in chemical reaction with heterogeneous catalysis, J. Chem. Phys. 56 (9) (1972), 4397-4400.

[13] C. V. PAO, Blowing-up of solution for a nonlocal reaction-diffusion problem in combustion theory, J. Math. Anal. Appl. 166 (1992), 591-600. 
[14] C. V. Pao, Nonlinear parabolic and elliptic equations, Plenum, New York, (1992).

[15] P. Quittner And P. Souplet, Superlinear Parabolic Problems: Blow-up, Global Existence and Steady states, Birkhauser Verlag AG, Berlin, (2007).

[16] M. Pedersen AND Z. G. Lin, Coupled diffusion systems with localized nonlinear reactions, Computers Math. with Appl. 42 (2001), 807-816.

[17] P. Souplet, Blow-up in nonlocal reaction-diffusion equations, SIAM J. Math. Anal. 29 (6) (1998), 1301-1334.

[18] P. Souplet, Uniform blow-up profiles and boundary behavior for diffusion equations with nonlocal nonlinear source, J. Diff. Equations 153 (1999), 374-406.

[19] L. W. WANG AND Q. Y. CHEN, The asymptotic behavior of blow-up solution of localized nonlinear equation, J. Math. Anal. Appl. 200 (1996), 315-321.

[20] M. X. WANG, Global existence and finite time blow up for a reaction-diffusion system, Z. Angew. Math. Phys. 51 (2000), 160-167.

[21] M. X. WANG, Blow-up rate estimates for semilinear parabolic systems, J. Diff. Equations 170 (2001), 317-324.

[22] Z. Y. XIANG, Q. CHEN AND C. L. MU, Blow-up properties for serval diffusion systems with localized source, ANZIAM J. 47 (2006), 37-56.

[23] L. Z. ZHAO, Singularity analysis of parabolic systems coupled via nonlinear inner sources and (or) boundary flux of exponential types, $\mathrm{PhD}$ Thesis, Dalian University of Technology (in Cainese) (2004).

[24] J. ZHOU AND C. L. MU, Uniform blow-up profiles and boundary layer for a parabolic system with localized sources, Nonlinear Analysis, 69 (2008), 24-34. 